\title{
Voyageurs occidentaux en Bulgarie et Roumanie
}

\section{Hélène Lenz}

\section{(2) OpenEdition}

\section{Journals}

Édition électronique

URL : https://journals.openedition.org/cher/8080

DOI : $10.4000 /$ cher.8080

ISSN : 2803-5992

\section{Éditeur}

Presses universitaires de Strasbourg

\section{Édition imprimée}

Date de publication : 1 décembre 2009

Pagination : 97-116

ISBN : 978-2-35410-006-3

ISSN : 1968-035X

Référence électronique

Hélène Lenz, « Voyageurs occidentaux en Bulgarie et Roumanie », reCHERches [En ligne], 3 | 2009, mis en ligne le 15 décembre 2021, consulté le 17 décembre 2021. URL : http://journals.openedition.org/ cher/8080 ; DOI : https://doi.org/10.4000/cher.8080

\section{(c) (i) (-)}

Ce(tte) œuvre est mise à disposition selon les termes de la Licence Creative Commons Attribution Pas d'Utilisation Commerciale - Partage dans les Mêmes Conditions 4.0 International. 


\title{
Voyageurs occidentaux en Bulgarie et Roumanie
}

\author{
HÉLÈNE LENZ \\ Université de Strasbourg
}

e réveil du Danube (1998) de Martin Graff récapitule des motifs présents une fin de communisme laissant envisager l'intégration européenne. Né en Alsace en 1944, auteur aussi d'un Voyage au jardin des frontières (2001), cet écrivain, enseignant, ancien pasteur, se présente comme un «connaisseur des réalités danubiennes». Il a remonté le fleuve pendant dix ans pour rendre compte des mutations de sa civilisation. Son livre - publié à Strasbourg - a obtenu le prix du journalisme franco-allemand. Parallèlement, il a donné lieu à une série télévisée récompensée par le prix du meilleur film économique ${ }^{1}$.

L'espace dévolu à sa «géopolitique vagabonde de l'Europe» coïncide avec l'aire culturelle envisagée vingt ans plus tôt par C. Magris («dictionnaire vivant du Danube»). Publié en Italie en 1986, en 1988 en français, Danube a été rédigé au début d'une Perestroïka perceptible dès 1982-1983 selon plusieurs observateurs. La cartographie du germaniste italien est proche à son tour de la Rhapsodie roumaine de D. Fernandez (1998) communiquant un état des lieux littéraire et artistique dix ans après la chute de Ceausescu, dix ans avant l'entrée dans l'Union Européenne. La même année, M. Graff insiste sur la géographie physique et humaine de l'ex-Mitteleuropa, sur la progression technologique de zones happées par l'économie de marché. Face à cette urgence, des actions sont engagées par des investisseurs allemands tandis que l'attitude française - non interventionniste - suscite son sarcasme. «Chaque lycéen français sait qu'Elias Canetti, prix Nobel de

1 Précisions de la jaquette, quatrième de couverture.

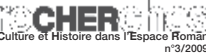


littérature, est né ici, au numéro 12 de l'Ulica Slavianska. Appartenir au peuple le plus cultivé de la terre est toujours rassurant» (Graff, 1998: 68). L'écriture diariste du Réveil du Danube en fait aussi un guide des mœurs au quotidien, censé introduire à la compréhension de pays encore sous le choc de changements idéologiques. Surtout fonctionnelle, la relation du périple emmagasine - tel un script se transformant en scénario - des réalités calibrées à l'usage des médias. Les concepts philosophiques régissant la culture danubienne du XIX ${ }^{\mathrm{e}}$ siècle ont été supplantés par la fascination de la mondialisation. Ici comme ailleurs, chacun s'approprie dès que possible les technologies modelant le réel et permettant d'en rendre compte. Le reportage/ essai/ journal de découverte est sans doute le mode livresque le plus approprié à des lecteurs convertis à une culture audio-visuelle de la «communication».

Selon les conventions réalistes dont relève aussi ce mode de représentation, des inconnus dialoguent, livrent à l'étranger les informations nécessaires à sa route, à la compréhension de leur mode de vie sans que le lecteur perçoive s'ils sont interviewés. L'impression de pléthore de projets, d'élans, l'euphorie communicative inhérente à ce trajet balkanique fait songer à une fête contemporaine d'hommes pressés. Elle rappelle aussi l'idéologie de l'authenticité à l'oeuvre dans les textes de Panaït Istrati (1884-1935) seul ex-journaliste roumain ayant conquis une gloire mondiale. En son temps aussi, l'auteur vagabond notait l'accélération du rythme des perceptions, la griserie des rencontres sans lendemain, l'ivresse paysagère propre au voyage, addiction culturellement valorisée.

A l'instar de Cendrars et de ses héritiers, M. Graff imprime au reportage un rythme lié au moyen de transport dont il use. Le poème-tableau était généré par le Transsibérien d'avant 1913. Un camion TV suscite une parenté du texte de Graff avec des narrations «sur la route» imitées de Kerouac, d'un style de films ( «road movies»), de récits d'excursions concomitants de la vulgarisation de la machine. Si le voyage se lie au vertige, depuis les futuristes italiens, des techniques prouvent que «l'automobile symbolise la vitesse en écriture mais aussi en peinture» (Monneyron et Thomas, 2005: 61). Le roman Flèche d'Orient (1932) de Paul Morand, inventeur de «l'homme pressé», "père du style moderne en littérature $»^{2}$ combinait déjà des focalisations typiques de: - la vue d'avion sur le trajet Paris-Bucarest, - du déplacement en voiture à l'intérieur de la capitale roumaine, - des

2 http://fr/wikipedia/org/wikiPaul_Morand, page consultée le 07/0/2009. 
méandres de la circulation à travers le Delta dans un rafiot de pêcheurs lipovènes.

Enfin, le cours du deuxième fleuve d'Europe après la Volga, en fonction duquel récit et trajet routier se positionnent, n'est jamais perdu de vue par le récit routier de $M$. Graff.

\section{Les brisées de Jules Verne suivies à rebours «On the road»}

Journal de bord d'un trajet reliant l'Ukraine à l'Allemagne, le Réveil $d u$ Danube voit dans la Bulgarie et la Roumanie deux étapes, deux nuances d'une opposition Orient-Occident transitant dans l'espace comme dans le temps entre slavisme et germanisme. Le Beau Danube jaune de Jules Verne ${ }^{3}$, avant Danube de C. Magris, cartographiait un espace allant de la source du fleuve (Donaueschingen, «marqué par une plaque») au Delta roumain donnant sur la mer Noire par trois bras (Magris, 1986; trad. 1988: 24, 540). M. Graff effectue le trajet en sens inverse. Le livre de Jules Verne comptait seize chapitres, le livre de Magris neuf, le reportage de Graff en a onze. Sa remontée du fleuve se détaille en: 1. Mise à flot, 2. Ukraine, 3. Moldavie, 4. Roumanie, 5. Bulgarie, 6. Serbie, 7. Croatie, 8. Hongrie, 9. Slovaquie, 10. Autriche, 11. Allemagne. La distribution du trajet fluvial sur dix pays (un tiers de sa longueur est en territoire roumain) est reflétée par le plan.

Sélectif, le périple de M. Graff ne pénètre pas l'intérieur des territoires. Les plages, les montagnes (Carpates et Balkans), les capitales bulgare, roumaine ne sont pas évoquées. En Roumanie, deux localités sont traversées: Galati, cinquième ville nationale, "Duisbourg du Danube dont les hauts-fourneaux enserrent la ville avec leurs mâchoires de feu» et Tulcea. L'équipe y résidera deux jours avant de se «remettre à rouler en silence vers le sud»: la Bulgarie. Dans l'intervalle, le Delta aujourd'hui classé réserve de la biosphère, zone

3 Le Beau Danube jaune fait partie du groupe de cinq romans remis à l'éditeur de J. Verne (J. Hetzel) après sa mort, après 1905. Hetzel a attribué au roman une date de rédaction: 1880. Dans l'édition Folio (2002), les seize chapitres découpés par les étapes du trajet fluvial portent les titres: I- Au concours de Sigmaringen. II- Aux sources du Danube. III- Une commission internationale. IV- Des sources du Danube à Ulm. V- D'Ulm à Ratisbonne. VI- De Ratisbonne à Passau. VII- De Passau à Lintz. VIII- De Lintz à Vienne. IX- À la sortie des Petites Karpates. X- De Vienne à Presbourg et Buda-Pest. XI- Kruschistes et antikruschistes. XII- De Pest à Belgrade. XIII- De Belgrade aux Portes de Fer. XIVNicopoli, Roustchouk, Silistrie. XV- De Silistrie à Galatz. XVI- De Galatz à la mer Noire. 
vedette d'un tourisme revu et corrigé par l'intégration européenne ${ }^{4}$, aura été vu.

\section{a. Le trajet routier}

Le trajet routier obéit à des exigences logistiques lourdes, fonction d'une stratégie professionnelle: filmer en temps limité. Le parcours rappelle une période déjà historique à l'époque de rédaction du texte. Au cours de l'après-89, des convois ont sillonné des territoires rescapés de révolutions sur des voies que l'Europe rénoverait bientôt à grand renfort de subventions, comme en témoigne "un ruban de macadam flambant neuf traversant un village » (Graff, 1998: 60). Cette volonté d'amélioration des communications se compare à l'arrivée du train au XIX ${ }^{\mathrm{e}}$ siècle dans la zone. Des pages de Léo Claretie (Claretie, 1913: 14) au roman d'Agatha Christie ${ }^{5}$, l'atmosphère de l'Orient Express est devenue un cliché artistique. En 1998, un tournage TV itinérant est encore une action fascinante - hors l'objet de son enquête relayée dans sa magie intrinsèque par l'évocation de ses conditions de déroulement.

Après l'entrée en Bulgarie, les véhicules traversant Silistra «ont foncé.» L'étape se confond avec l'hôtel Riga d'où l'équipe rayonnera avant un arrêt à Ruse (Graff, 1998: 65-69). Ce Roustchouk de Canetti - $495 \mathrm{~km} \mathrm{du}$ parcours - est brossé dans sa métamorphose consécutive à la chute du Mur de Berlin (le narrateur n'a pas revu la ville depuis sept ans). Des rencontres emblématiques de la synthèse entre ancienne et nouvelle société marquent le lieu: un millionnaire du loto bulgare, un philosophe au chômage, marié à une Anglaise, lisant Wittgenstein. Le philosophe est buraliste. Nul commentaire ne relève la production de tabac bulgare: une des premières d'Europe. L'ouvrage de M. Graff en effet se comporte en guide touristique par accès, sur des thèmes relevant d'un choix d'auteur.

Près de Volgrad, $503 \mathrm{~km}$, l'équipe du réalisateur profite du beau temps pour filmer le «fameux pont de l'Amitié» reliant Ruse à Giurgiu en

4 The Danube Delta, 2008: 24-25. Hors des circuits familiarisant le visiteur avec la culture et l'histoire de la région, les principales formes du tourisme sont: - relaxation and recreation - knowledge tourism (itinerant) - specialised tourism - scientific (destiné aux ornithologues, chercheurs, étudiants) - special youth programmes (apprentissage du contact avec la nature) - rural tourism (favorisant le contact avec les ressources locales: guides locaux, séjour chez l'habitant) - nautical sports and photo-safaris - sport fishing (sport hunting).

5 Le crime de l'Orient-Express d'A. Christie a été publié en 1934. 
Roumanie. «L'amitié obligatoire» d'autrefois - époque communiste - est sans doute en voie de se modeler sur le couple "franco-allemand». Ses relations ne tarderont pas à se transformer en "partenariat économique et politique »(Graff, 1998: 72).

Mouvementé, le parcours roumain esquisse une sociologie du déplacement routier intégrant plusieurs modalités de l'accident: «drame, épreuve, écueil du récit automobilistique» (Monneyron et Thomas, 1998: 60). Près de Galati, «un grand père est éjecté du bus que les passagers continuent à prendre d'assaut par vagues successives comme pour forcer un destin qui les a souvent oubliés»(Graff, 1998: 39-45). Plus loin, une jeune femme en Dacia enfonce «la porte droite avant d'une Mercedes appartenant à un Roumain exilé en Allemagne depuis trente ans ». Le propriétaire de la berline prend le cinéaste à témoin: une porte de Mercedes vaut dix mille francs. Des remarques sur la responsabilité, le destin ponctuent la confrontation entre «exilé devenu riche et Roumaine restée au pays». Tandis que l'équipe mène son train, des flashes captent des scènes de genre. "De jeunes Tziganes se chargent de nettoyer les vitres des voitures» et se battent pour partager le salaire. Une voiture coréenne achetée par un couple à Craiova n'a pas « une égratignure». Le samedi soir, dans le hurlement des hauts-parleurs versant leur musique sur les villages, les phares de l'équipe «happent le rire nerveux d'adolescentes». La poussière, topos littéraire et pictural roumain dès le $\mathrm{XIX}^{\mathrm{e}}$ siècle, est cadrée dans un nouvel avatar. "Même en roulant doucement, nous soulevons la poussière de la steppe de Baragan comme dans un film de pub.» Conducteur secourable (il a récemment convoyé un Roumain égaré sur la bande d'urgence de l'autoroute Paris-Strasbourg), le narrateur est bouleversé par une double tragédie: accident de personne et non assistance à personne en danger. Des chauffards n'ont pas stoppé après avoir renversé un jeune motocycliste sur une ligne droite. "Je m'arrête à sa hauteur. Il est couché surle dos et se relève, le visage ensanglanté [...] Quelques jours plus tard, une voiture avec un cercueil en bois sur le toit nous croisera.» Si en Occident, l'automobile est simple baromètre des statuts économiques, elle reste ici le reflet d'aspirations moins mesquines. Joro, en Bulgarie, a deux voitures: une vieille Volkswagen pour ses déplacements et une BMW ne roulant pas: «décapotable pour ses rêves». Ainsi dans La Grimace de Heinrich Böll, le héros fait tourner à vide les roues d'un véhicule arrêté pour lui faire atteindre le kilométrage obligatoire (Graff, 1998: 80). Averti de la référence littéraire par le reporter, Joro se débarrasse d'un véhicule. En Roumanie, l'automobile sert de test aux dérives de la sociabilité. Le narrateur 
a retrouvé deux amis techniciens autos sous Ceaucescu, reconvertis depuis 1992 dans une entreprise de recyclage. Leur fils écologiste - sa Dacia a été rayée par une clé malveillante - émet le constat: «Les gens n'ont aucune culture ici!» (Graff, 1998: 67). Le fait se confirme sur la route de Braila: des Tziganes en attelage y dépassent camions, voitures échouées. "Il n'existe pas ici de Touring Club vous dépannant en deux minutes. À chacun de se débrouiller pour conserver son moteur et son travail.» Peut-être, dans trois heures ou le lendemain, un chauffeur aura-t-il fait redémarrer son bus. «Entre-temps, ses passagers seront déjà repartis à pied.»(Graff, 1998: 57). Topos du récit de panne en zone climatique défavorisée, le détail rappelle aussi la voiture d'un journaliste français de l'entre-deux-guerres refusant de «lutter de vitesse avec les traineaux sur la route des isbas aux toits de chaume». Quand elle rend l'âme, son chauffeur occidental «soulève son couvre-oreilles pour écouter le fragile cœur d'acier balbutier sa peine» (Bourcier, 1932: 12-13). De toute évidence, les Balkans n'ont toujours pas les moyens d'assumer la croissance de leur parc automobile.

Face à l'incompétence mécanicienne, aux avaries prévisibles, la Bulgarie est un parangon de prévention. Elle "est le seul pays danubien imposant à ses visiteurs la purification des pneus et du bas de caisse de leur voiture. Après avoir sacrifié à ce rite, nous serons autorisés à fouler le territoire». Pourtant c'est en Bulgarie que deux voitures percutent de nuit un rouleaucompresseur sous les yeux du narrateur roulant en tête du convoi: il n'y a pas un survivant (Graff, 1998: 65).

\section{b. Parcours fluvial : jeux d'eau}

Le lyrisme brut de lieux sans artifice est revivifié par des métaphores néo-romantiques. L'ampleur des dimensions du flux rappelle-t-elle Johann Strauss, Smetana? Près de Tulcea, des «centaines d'amoureux sniffent le Danube». (Graff, 1998: 46-82). Ruse, "petite Vienne», dispense l'illusion de se trouver en Autriche ou Allemagne. Ses cafés ont des terrasses, ses rues sont piétonnes, ses habitants ont un air de «Prussiens, décontractés, cool». Une école privée nommée «Europe» a des locaux «ayant vue sur le Danube». Sur la route, une «longue vue imprenable» s'offre aux voyageurs (Graff, 1998: 76). Elle serpente sur des falaises «à hauteur de Nice.» Des langues de sable parsèment le fleuve. Pour cause d'embargo consécutif à la guerre de Yougoslavie, nul navire en vue, mais on voit toujours la Roumanie en face. Vidin s'enorgueillit de disposer du seul théâtre balkanique donnant sur les eaux. Grâce à un Allemand propriétaire de quatre mille véhicules: 
«Les camions arrivent de Passau sur d'énormes barges à raison de soixante unités par barge. Les chauffeurs bulgares les prennent en main, direction Proche-Orient ». À l'ombre de la forteresse de Baba Vida, un nageur émerge pour témoigner en allemand de son cosmopolitisme (Graff, 1998: 82). Enfin «une grande virée trans-danubienne» en paquebot au large de l'île de Belene rappelle une croisière de huit jours passés à manger, boire, dormir sans que les «touristes s'intéressent à leur voyage» avant Belgrade et Budapest. L'hymne national bulgare comparant ce pays au paradis célèbre d'ailleurs aussi le Danube (Graff, 1998: 69-74).

En Roumanie, on rejoint le bras de Sulina. Le prochain pont se trouvant à $253 \mathrm{~km}$ (Hirsova), on emprunte le bac. Une aube de septembre sur Tulcea découvre l'attrait esthétique d'un paysage industriel telle une pochette pour disque pop des années 70 cadrant un décor anglais. "La brume flotte sur l'eau. Un tanker passe comme un fantôme. Les hauts fourneaux crachent leurs saletés acides. Le mélange avec la condensation naturelle des eaux du fleuve percées par le soleil levant fait revivre la création du monde» (Graff, 1998: 46- 53). Le même jour, Felicia, mère du jeune Virgil à la Dacia rayée, déclame face à la caméra un poème de Heine, d'habitude écouté par les visiteurs du Rhin devant la Lorelei, rocher mythique de la fée aux pêcheurs. À Murighol (Delta), on loue une barque. Mais à l'inverse du héros de P. Morand, de C. Magris, de D. Fernandez, l'auteur du Réveil du Danube ne vague pas où bon lui semble. En effet, «les nouveaux écolos sont partout.» Le monastère de Saon, au bord du lac de Rotuna relié au Danube, «montre la coupole d'argent de l'église scintillant au soleil. De loin elle semble posée sur l'eau. Le monastère est plus facilement joignable par voie lacustre que par voie terrienne.» À Braïla, «en dépit des remontrances des capitaines, les gosses jouent à plonger dans le Danube» tels les compagnons suicidaires de Nerrantsoula dans la nouvelle éponyme d'Istrati. La notation précède des lignes évoquant l'apport de l'écrivain franco-roumain, un des rares auteurs signalés par M. Graff.

En Roumanie toujours, un autochtone réclame un gros plan. Ce pêcheur lipovène, "à béquilles, parle comme un poète». Il va crier "Ceausescu» devant le fleuve en tapant avec une de ses béquilles sur la tête d'un serpent noir qui tente de se sauver sous les eaux: "Ceauşescu, Ceauşescu! Camera, please!»

Armin est déjà au travail mais les cris du paralytique ont réveillé Horst, directeur de la photo. Ce dernier déboule en courant, arrache la caméra des mains de son assistant, poursuit le serpent dans le Danube sans voir 
qu'il a déjà de l'eau jusqu'à la ceinture. Mioara m'éclaire: la rumeur veut que Ceausescu se réincarne dans les serpents du fleuve pour être parmi nous. - 'Un plan extraordinaire' s'exclame Horst en sortant enfin de l'eau. (Graff, 1998: 63)

\section{c. Vélos de luxe}

Le trajet roumain parallèle au fleuve notait la présence d'arbres comme on en voit dans les promenades au ralenti. Avant Ruse

des milliers de noyers jalonnent la route [...] Cerisiers au printemps, noyers en automne, ces nuées d'arbres nourrissent ceux qui ont faim. Ils appartiennent à tout le monde, comme les puits permettant au voyageur de calmer sa soif. Ils ressemblent à des mâts de voiliers abandonnés dans la nature. On les actionne pour faire remonter le seau des profondeurs de la terre. Nous préférons pourtant l'eau minérale 'Izvorul Minimilor, compoziție chimică după Fresenius, Germania'. Encore un marché qui a échappé à la France au profit de l'Allemagne. (Graff, 1998: 57)

L'équipe croise "un Martien et une Martienne»: deux étudiants cyclistes excitant la curiosité villageoise bulgare. On vient les toucher tant ils semblent surnaturels avant que des gamins hostiles ne les bombardent de pommes.

Encore un paradoxe danubien: ce n'est pas en voiture qu'on se fait remarquer, c'est en vélo que l'on ressemble à un extraterrestre. Ce jeune couple croyait bien faire en se fondant dans la nature [...]. C'est à vélo qu'il attirera l'attention [...] parce que le vélo reste une denrée rare en Bulgarie, mais (aussi) parce que le modèle est perçu comme le représentant de la haute technologie d'Occident. Le vélo comme symbole du luxe, même les Verts allemands n'y auraient pas pensé. (Graff, 1998: 78)

\section{Un parcours germanophile}

Le collectage d'informations filmiques prédéterminait la finalité de l'expédition, ses étapes. Une commande de ZDF, deuxième chaîne TV allemande, a décidé en effet du départ de Graff pour l'autre bout du fleuve, en 1997. L'auteur réalisateur de la série est «seul Français parmi quatre Allemands qu'il énerve avec ses idées d'Alsacien casse-frontières» (Graff, 1998: 11). Ses co-équipiers sont des techniciens. Les autochtones les apprécient pour des qualités dont nul ne dispute la suprématie à des Allemands en Europe: efficience, maitrise technique. Le crédit dont ils jouissent ici se confond avec l'aura de leurs compétences. Riches, ils logent dans les palaces offrant un repos mérité à leur productivité, à leur réactivité, 
au savoir faire élitaire dont elle résulte. «Le reportage requiert prestige et élégance» soulignait déjà la préface de Reportages des années 1930 réédités cinquante ans plus tard à Bucarest (Brunea-Fox, 1979: 37). L'élégance moderne est peu salonarde. Jeunesse et allure sport conféraient à Filip Brunea-Fox, "prince du reportage roumain», son titre, décerné en 1928 par Gheorghe Dinu dans Unu, revue de l'avant-garde, «d'ange cow-boy.»

Trois équipiers de M. Graff sont des hommes. Quoique né près du Rhin, Horst le cameraman est «très prussien ». Armin, "son assistant beau-gosse, est spécialiste des caméras high tech». Michael, chargé du son, est un «écolo puritain abreuvé d'eau minérale». Monika, seule femme de l'équipe, «organise le tournage en s'occupant des policiers les plus retors». Elle a été top-model en RDA. Par elle, familiarité avec l'univers professionnel de la photogénie et connaissance innée des interdits communistes / postcommunistes à ne pas transgresser s'ajoutent aux atouts diplomatiques du groupe (Graff, 1998: 11).

\section{a. «balkanique » et « scandinave»}

Voyant dans l'emploi du terme «balkanique» un défi à relever, C. Magris comparait les mœurs yougoslaves, bulgares à celles de nations exemplaires.

Balkanique [...] relève du vocabulaire de l'insulte [...]. Mais quiconque ayant vu les rues de Sarajevo et de son bazar, nettes comme un miroir, ou la propreté impeccable de Sofia, les compare à ces villes, à ces États cités $[\ldots]$ comme des modèles de civilisation, est enclin à employer le terme 'balkanique' comme un compliment, comme d'autres ont l'habitude de dire 'scandinave'. (Magris, 1998: 471)

\section{b. Bulgarie et origine du germanisme}

À la suite de Magris encore, Graff situe en Bulgarie les sources du germanisme. Si son appartenance "alsako-bochique» (sic) - déterminante dans son contrat avec la ZDF - le rend attentif aux formes réprimées et résurgences d'une domination dont il est partie prenante en qualité de Français, il n'en évalue pas moins en professionnel (puisqu'il est pasteur) les causes d'un succès moral impliquant jusqu'à l'Amérique. La Bible a joué un rôle dans le destin germanique. La force allemande inséparable du puritanisme luthérien est aussi à l'origine de l'Europe.

Magris signale que de ces rives est parti le germanisme il y a dix siècles. L'évêque Wulfila, arrivé avec les Goths, avait traduit la Bible en gothique. Je 
n'avais jamais fait le rapport entre Luther et le Danube. Imaginez l'Europe sans Luther... Que se serait-il passé si l'évêque Wulfila s'était noyé dans le Danube à Nikopol? (Graff, 1998: 77).

\section{c. Hommes d'affaires en «Europe» ex-communiste}

Un grand nombre d'Allemands sont rencontrés au quotidien par les voyageurs. Le dynamisme postcommuniste bulgare a transformé le pays en colonie:

Les Allemands sont partout. Je croise plusieurs camions de la Croix-Rouge à destination de l'ex-Yougoslavie qui font l'objet de tracasseries administratives. A l'entrée de Vidant, un motel a été construit par un Allemand marié à une Bulgare. Une grande usine de chemises a été acquise par des industriels d'outre-Rhin. Ils viennent de célébrer le trentenaire de l'entreprise, y incluant généreusement la période communiste. Mais l'Allemand le plus célèbre de Vidin sinon du Danube est sans conteste Willy. Betz de son nom de famille. (Graff, 1998: 82)

L'industriel est propriétaire de quatre mille camions. M. Graff loue son action en termes évocateurs de son propre choix de civilisation: «ce qu'on appelle un ' joint venture' écolo et efficace». Son livre insistera souvent sur le rôle crucial de l'écologie dans des pays où le communisme a pratiqué, aux côtés du gaspillage d'énergie humaine, une destruction de l'environnement passée sous silence jusqu'à la fin (Graff, 1998: 66-82).

Un Autrichien construit des liens industriels. Comme l'écologique Willy Betz, il exerce son activité dans un souci de transition attentif à prévenir les heurts. Nombre de ses contacts datent du communisme. Il connaît les lacunes du nouvel Est en matière de formation spécialisée. Confronté au problème de l'éducation, M. Graff notera l'impossibilité pour les étudiants du Sud-Est d'obtenir des visas permettant l'accès aux universités occidentales.

Ehrhard Brosenbauer est autrichien, de Linz, la ville danubienne dont Hitler voulait faire la capitale du Reich. L'ingénieur travaille comme consultant dans l'industrie. Son rôle est de créer des 'joint ventures' bulgares avec les entreprises occidentales [...] 'Ce sont les écoles pour managers qui manquent. Il n'y a pas assez de cadres. L'enthousiasme ne suffit pas' me dit l'homme d'affaires autrichien. (Graff, 1998: 66)

Un autre Willy - 75 ans - raconte ses souvenirs de la SS sur le bateau à vapeur du Danube. Il a été emprisonné par les Russes, par les Américains. Libéré, il est devenu un «modeste banquier bavarois». Pendant la guerre, Willy a apprécié les alliés bulgares. 
Il m'explique qu'il a traversé le Danube avec sa compagnie SS en hiver 1941 près d'ici en direction de la Grèce. Je lui demande naïvement comment c'était. "Ah! la guerre des Balkans a été une guerre joyeuse (ein lustiger Krieg). Mon étonnement ne le rend que plus loquace.' Oui, joyeuse, d'ailleurs les gens nous ont accueillis en héros et nous avons festoyé ici comme des dieux’». (Graff, 1998: 84)

Le pragmatisme allemand est corollaire d'un idéalisme actif. Il s'oppose à une rhétorique française dérisoire de narcissisme, méprisable même.

Au petit déjeuner, j'observe le manège des industriels allemands qui font des affaires tandis que les stratèges parisiens genre Alain Minc s'époumonent sur les plateaux télévisés. Tenez: le docteur Maurer, ex-secrétaire d'Etat à Bonn spécialiste en agriculture, s'entretient avec Gradiu Chadschik, le patron des laiteries de la région de Ruse. Il est à la retraite et pourrait se contenter de boire son petit vin blanc en rêvassant le long du Rhin à Mayence où il habite. Mais il a décidé de partager son savoir. Vous imaginez sincèrement un ex-ministre ou secrétaire d'Etat énarco-normalien sinon polytechnicien, s'abaisser à voyager le long du Danube pour conseiller ses frères européens? (Graff, 1998: 67)

En Roumanie aussi, les «voisins d'outre-Rhin sont déjà présents sur le terrain ». Le premier Occidental rencontré à Galati, Josef Bentfeld, construit une usine à produire de l'huile (Graff, 1998: 42).

\section{d. Une influence germanique ancienne}

La Roumanie confronte de manière unique culture allemande et culture latine, observe M. Graff. Le point de vue n'est pas nouveau. Soutenu entre les deux guerres mondiales par B. Fondane - écho de son temps - à propos de critique culturelle, c'est aujourd'hui un cliché européen. La Transylvanie fut peuplée de Saxons dès le XII ${ }^{\mathrm{e}}$ siècle. Le Banat a accueilli des colons de Souabe, d'Alsace-Lorraine au XVIII ${ }^{\mathrm{e}}$ siècle. Ces faits donnent lieu à des observations d'histoire des mentalités que le style vulgarisateur humoristique de l'ouvrage dédouane de l'accusation de banalité. "Comment vivaient au quotidien ces vaillants Germains? Quel style ont-ils donné à leurs maisons? Qu'ont-ils bu et mangé?» L'observation sur les guerres franco-allemandes, susceptibles d'avoir été évitées si quelqu'un avait accepté de "pratiquer une étude approfondie des relations germano-roumaines » souligne l'importance accordée par l'auteur à une présence germanique cruciale pour la zone et le monde (Graff, 1998: 42).

La question de l'éducation des élites est nodale aussi pour qui envisage la concurrence d'influences en Europe, comme le montrent d'anciens 
voyageurs. Supériorité incontestée, pressions colonisatrices? T. Todorov confortant la réputation de l'éducation germanique souligne de fait les affinités de la Bulgarie avec l'esprit de sérieux. Son pays de naissance

n’a pas eu dans le passé de rapports suivis avec la France. Des liens traditionnels existaient avec la Russie, l'Allemagne [...] géographiquement plus proche de la Bulgarie [...] mêlée de plus près à son histoire; nos rois, jusqu'à la fin de la Seconde Guerre mondiale, étaient des 'Saxe-CobourgGotha'[...]. C'est en Autriche ou Allemagne que partaient avant la guerre les fils (plus rarement les filles) de bonne famille, quand ils voulaient faire des études ou plus simplement 'voir l'Europe'. Des artistes, des savants étaient bien passés par la France (Nancy, je crois) mais ils n’étaient pas forcément les plus estimés: l'éducation germanique avait la réputation d'être plus solide. (Todorov, 1996: 235-236)

De fait, les rois roumains aussi étaient allemands. En 1913, Léo Claretie consacrait un chapitre à l'éloge de leurs activités dans ses Feuilles de route (Claretie, 1913: 18-44). "Le roi Carol Ier est un Hohenzollern. La reine Elisabeth de Wied [...] est allemande. Le prince héritier, neveu de roi, a épousé une princesse de Saxe- Cobourg Gotha.» La reine (en littérature Carmen Silva - sic -) joue un rôle dans la diffusion de la culture française entre les deux guerres mondiales. Elle en maîtrise la langue au même titre que l'allemand. Elle converse en français avec le journaliste l'interrogeant sur son dernier livre (les «Contes de Pelesch»), sur un hobby de folkloriste inséparable de sa fonction (elle a recueilli auprès de paysans légendes allemandes et roumaines). Son cabinet de travail encombré aussi de partitions témoigne d' «un labeur assidu». Quant au bureau «simple, sobre» du roi, «c'est la table d'un soldat, d'un penseur, d'un politique sage ». La famille royale est impliquée dans l'éducation féminine, la valorisation d'activités tenant de l'industrie et de l'art: ainsi l'artisanat textile populaire. L'attachement aux traditions n'empêche pas une exemplarité de comportement ultramoderne: anglo-saxonne. Dès 1913, la reine Elisabeth écrivain «use de la dactylographie». La princesse Marie «très sportive, colonelle d'un régiment de hussards, galope à sa tête, hardie à l'obstacle et dure à la pratique.» L'affabilité royale témoigne d'une absence d'affectation démocratique. La France occupe dans la réflexion des souverains germaniques une présence prouvée par la présence dans leurs bibliothèques d'une majorité de textes français. Ne pratiquant ni faste déplacé, ni frivolité, la Cour est exemplaire de vertus civilisatrices dans un pays trop récent.

En Roumanie, la Cour est plus grave que la ville. La nation est gaie, remuante, amie du plaisir: les souverains tempèrent ces juvéniles ardeurs d'un 
peuple de trente ans. Le char a des coursiers ardents et un aurige prudent. (Claretie, 1913: 25)

Un partage préférentiel des influences (allemande en Bulgarie, française en Roumanie) s'est-il effectué sur décision politique? Si L. Claretie suggère qu'une infiltration française en Bulgarie aurait pu être légitimée par l'origine de personnes royales puisque «le prince Ferdinand, un Saxe-CobourgGotha, est né d'une mère française, Clémentine de Bourbon-Orléans», il considère, par conviction préalable ou acquise sur le terrain que:

Les jeunes gens (roumains) faisant leurs études en France rapportent une qualité d'esprit supérieure à celle des étudiants revenant d'Allemagne, parce que ceux-ci ont grandi au milieu d'une race qui leur était étrangère. Ils sont liés à nous par la reconnaissance, par le souvenir de ce que la cause de l'indépendance doit à Napoléon III, à Edgard Quinet, à Michelet, à Mme Cornut. Et nous les aimons pour leur vaillance héréditaire. Au Xve siècle, ils furent le rempart de l'Europe contre les Turcs. (Claretie, 1913:16-18)

Les détails du passé roumain et bulgare - différents de la situation grecque ayant connu des rois allemands mais non le communisme, à l'inverse de l'Est de l'Allemagne - justifient aussi les interrogations de M. Graff. La distribution d'influences en Europe centrale le renvoie à un questionnement sur une identité alsacienne au coeur d'une expérience sur les causes, les effets d'une interaction franco-germanique présentée comme éprouvée personnellement.

\section{Mohicans d'Europe et lieux interdits}

La pierre d'achoppement de la supériorité germanique demeure le nazisme et son corollaire: l'antisémitisme. Le communisme a assis sa domination sur la condamnation du premier sans entreprendre le blâme en profondeur du second. M. Graff évite le piège tendu au germanophile en pointant du doigt un crime passé sous silence durant le communisme mais dénoncé en 1946 dans ses «horreurs inqualifiables» par une chronique française sur Bukovine et Bessarabie (Blairy, 1946: 213-217). M. Graff suggère aussi l'identité des vrais complices.

Les Roumains qui s'appelaient encore Valaques au XIX ${ }^{\mathrm{e}}$ siècle, crient leurs origines latines sur tous les toits du monde. Ce qui ne les a pas empêchés de cultiver leur antisémitisme et de flirter un peu trop intensément avec Hitler, tout comme l'Italie et la France. (Graff, 1998: 42) 
Le titre de fierté bulgare en la matière: avoir refusé la déportation en dépit du pacte passé avec Hitler, ne figure pas dans le chapitre de M. Graff consacré à la Bulgarie. La fragilité du bien, l'ouvrage de T. Todorov sur le sauvetage des juifs bulgares, a été publié en France un an plus tard (1999).

Les haines nationales réactivées en 1998 sont déplorées en termes proches du style rousseauiste de José d'Orient, voyageur français affirmant en 1932 son attachement à ses origines grecques.

Au pays d'Ésope - mon pays! - l'on devrait pouvoir comprendre mieux qu'ailleurs de quels méfaits la langue peut se rendre coupable. [...] J'aimerais voir bannir du langage ces distinctions de Juif et de Grec, aussi bien que de Turc, de Bulgare, de Tzigane, d'Albanais ou de Roumain, par quoi se perpétuent les barrières dressées par les siècles entre les éléments fraternels! ... (D’Orient, 1932: 134-135)

\section{a. Retour du religieux}

L'aspiration à une liberté sacrée est enthousiasmante. Cependant, cette liberté étant aujourd'hui défendue par des popes, les Roumains gagneraient à introduire «un zeste de laïcité» dans leur vision sociale, observe M. Graff. C'est dans le chapitre sur la Roumanie que la multi-culturalité balkanique est commentée dans l'évocation des craintes démocratiques qu'elle inspire à un citoyen français. Des nations voisines se méprisent par tradition nationale au moins tolérée durant le communisme.

Avant la chute des murs, lorsque j'ai commencé une collaboration avec les télévisions des pays danubiens, c'était la même rengaine [...]. Le Roumain était un barbare aux yeux du Bulgare. Le Bulgare aux yeux du Roumain. Le Serbe était un barbare aux yeux de tous. Il suffirait d'un mariage malheureux entre un Le Pen danubien et une crise économique mondiale pour enflammer le Danube. (Graff, 1998: 51)

Les pays avancés du globe expérimentent aujourd'hui la cohabitation interculturelle. Or, dans l'Europe orientale revisitée par M. Graff, coexistent de fait deux états de civilisation. D'une part, une situation linguistique d'apparence bénigne décrite par un journaliste français déjà cité.

Pour distinguer les multiples représentants des races échouées sur cette bande de terre [...] faute de bien saisir leurs noms variés et bizarres autant que par paresse d'esprit, on a accoutumé de dire: le Grec, le Juif, le Turc, l'Albanais, le Roumain, le Tzigane, le Bulgare... (D’Orient, 1932: 131)

D'autre part, le parallèle européen d'une «mixophobie» américaine contemporaine montrée comme effet de l'institutionnalisation de groupes 
à l'intérieur de nations niant les "valeurs démocratiques fondamentales». (Todorov, 1996: 219-221). Ces groupes insistent «sur l'appartenance d'origine plutôt que sur l'accomplissement de l'individu. Ils contribuent de ce fait à l'épanouissement de 'l'orgueil ethnique' comme le dit ingénument le président d'une université des environs de Boston». On peut considérer que le parti-pris économique de Martin Graff se donne pour fonction (très française) de situer les problèmes politiques et sociaux sous le signe d'une mondialisation nivelant des appartenances ethniques historiques meurtrières ou mortelles. Des guerres rapprochées ayant fait fluctuer les limites territoriales, les pays d'Europe de l'Est enregistrent l'existence de minorités réciproques puisant dans la fin du communisme une force d'identification accrue aux origines. Ainsi Zatouchnitza, fête de Valaques bulgarisés célébrée à la campagne, honore les morts avec une ferveur transformant l'église en:

caverne d'Ali-Baba sortie de l'imagination de Steven Spielberg. Des centaines de bouteilles de vin et de jus de fruits garnissent les tables et le sol. Des milliers de bougies, coincées directement dans les gâteaux, se reflètent dans les icônes ornées de crêpes noirs. (Graff, 1998: 85)

Si le voyageur occidental esquisse la description d'une coutume postcommuniste avec la sérénité d'un reporter averti de l'existence de pratiques plus pittoresques encore chez les Mexicains, le sociologue P.H. Stahl, d'origine roumaine, envisage le phénomène sous l'angle d'un retour du religieux susceptible d'impliquer l'ensemble de l'Asie. La religion, nœud du sentiment d'identité, a été éradiquée dans le Tibet assujetti par la Chine, dans les pays baltes, la moitié occidentale de la Pologne, la Bucovine et Bessarabie roumaines écrasées par la Russie.

Dès l'instant où le pouvoir communiste installé dans les pays de l'Est européen commence à s'estomper, des réalités profondes venues du fond des âges [...] reparaissent. Empêchées de se manifester par un pouvoir dictatorial, les appartenances ethniques et religieuses ressurgissent d'autant plus violemment qu'elles avaient été plus fortement compressées. (Stahl, 2004: 55)

\section{b. Suites d'une phobie anti-turque}

Evoquée par L. Claretie comme un titre de gloire historique roumain, la vigilance anti-turque - puisant sa principale justification dans un refus de la domination ottomane - est surtout évoquée par $\mathrm{M}$. Graff en Bulgarie où les fantasmes sur le thème frisent le seuil mythologique. Les médias jouent un rôle non négligeable dans la réactualisation romancée d'une histoire 
nationale d'ailleurs partagée avec les Roumains (comme en témoigne l'œuvre de Virgil Gheorghiu - 1916-1992 - riche en anecdotes sur les horreurs de l'Occupation). Ainsi Monika, l'assistante du narrateur, actrice de cinéma en RDA vingt-cinq ans plus tôt, a un ami bulgare "passant précisément à la télé» lors de l'arrivée de l'équipe.

Il y joue le rôle d'un commandant turc, né bulgare sans le savoir, enlevé bébé par l'armée turque pour se voir élevé dans la plus pure tradition des janissaires, le corps d'élite ottoman. Adulte, il revient dans son pays opprimer son propre peuple, chargé de capturer les enfants bulgares qui seront élevés en Turquie, avant de revenir en conquérants dans leur patrie opprimée. Victimes du célèbre 'coma des racines', ils opprimeront les leurs avec une violence décuplée par l'inconscient. (Graff, 1998: 68)

Vieille connaissance de 1990: «George, mon contact à la télévision bulgare affirmait le plus sérieusement du monde (à l'époque): les infirmières turques (bulgares) piquent les patients bulgares pour les rendre impuissants. »Une autre familière du narrateur, nommée Dilek, est «princesse». Sa généalogie l'autorise-t-elle à pratiquer l'investigation raciale?

Elle prétend sans hésitation que les Bulgares sont des Turcs. Une réponse se trouve peut-être dans la tentative d'assimilation par les Bulgares de la minorité turque, près de huit cent mille personnes [...]. Et si tous les Bulgares étaient des Turcs christianisés? me demande ma princesse. Mes protestations sont d'avance vouées à l'échec. (Graff, 1998: 68)

De fait en Bulgarie, en matière de Turcs (le point ethnique sensible par excellence), tout est affaire de bonne grâce dans l'examen du problème. Ainsi, à Vidin, de nouvelles dispositions architecturales annoncent une volonté de coopération aujourd'hui vérifiée: des sondages de 2007 révèlent que les Bulgares sont partisans d'une entrée de la Turquie dans l'Union Européenne.

Même les Turcs semblent avoir laissé un bon souvenir à Vidin. Le pacha Osman Pazvantoglou fit mettre un cœur à la place du croissant au sommet de la mosquée. Il était né de père turc et de mère chrétienne. Par ce geste symbolique, il voulait démontrer la nécessité de la coexistence pacifique entre les cultures. L'édifice est en train d'être rénové, de même que la synagogue. (Graff, 1998: 68-73)

Les haïdouks remémorés par Christo Botev, héros anti-ottomans (et anti-islamiques) justiciers du peuple sont-ils désavoués après deux siècles de gloire populaire littéraire roumaine et bulgare? C. Magris l'envisage: "Georg Rosen, auteur d'ouvrages sur les Haïdouks et traducteur de leur poésie populaire, se demandait si leur incessante guérilla avait défendu 
la nation bulgare ou retardé son développement économique» (Magris, 1988: 471). De nouveaux accords avec un Etat turc moderne négationniste du génocide arménien seraient-ils justifiés par le marasme financier? Monika et Laura, trente ans, à la fois bulgare et arménienne - «nous sommes en pleine multiculturalité» - sont invitées à s'exprimer sur une rumeur: "est-il vrai que des enfants meurent de faim dans (leur) pays?» (Graff, 1998: 73). Défendant l'essor économique de son pays dans sa vie professionnelle, Laura n'est au service ni des Turcs ni des Allemands. Elle est employée par un mécène surgi au cours de cette période: "la fondation de Georges Soroș, le milliardaire américain d'origine hongroise qui fait chuter la Bourse quand il tousse et investit énormément dans les ex-pays communistes» (Graff, 1998: 70).

\section{c. Lieux interdits}

Sur l'intervention de Laura, le Français obtient l'autorisation de filmer un lieu signalé par T.Todorov évoquant le système concentrationnaire bulgare: l'effrayante prison de Béléné, analogue d'Auchswitz, équivalent de la prison roumaine de Sighet devenu Mémorial des Victimes du Communisme ${ }^{6}$ et réplique des camps évoqués par Soljenitsyne dans L'Archipel du Goulag.

On connaissait [...] l'existence d'un camp de travail, une colonie pénitentiaire, en Bulgarie même, à Béléné, sur une île au milieu du Danube. Béléné était même devenu comme un nom commun de la langue bulgare de l'époque. On disait 'Tu vas finir à Béléné 'à chaque personne qui avait un comportement sortant des normes, d'une manière ou d'une autre - parce qu'elle s'habillait de façon excentrique ou qu'elle racontait un peu trop d'anecdotes anticommunistes. [...] Il existait un lieu où l'on enfermait les anormaux du point de vue psychique, la 'maison des fous' à Karloukovtsi et un lieu où l’on enfermait les anormaux sur le plan social, Béléné. C'était 'normal'... (Todorov, 2002: 277-278)

Commentant l'apparente modification du système carcéral consécutive aux révolutions, $\mathrm{M}$. Graff anticipe de fait les observations de Todorov:

L'île ne possède aucun système de sécurité visible et les prisonniers travaillent dans les champs comme des ouvriers ordinaires. [...] Lorsque les touristes remontent le Danube en paquebot, ils longent la très belle île de Béléné, $\mathrm{km} \mathrm{570,} \mathrm{sans} \mathrm{savoir} \mathrm{qu'ils} \mathrm{passent} \mathrm{à} \mathrm{quelques} \mathrm{mètres} \mathrm{de} \mathrm{la} \mathrm{plus} \mathrm{grande}$ prison bulgare. Ce n'est pas surprenant. La grande virée trans-danubienne

6 Fondé par l'Académie Civique et en particulier par les écrivains Ana Blandiana et Romulus Rusan. 
est l'exemple même du tourisme aveugle. Chaque guide parle le drapeau national à la main, pérorant avec nostalgie sur un passé mythique. Les touristes ne sauront donc jamais que Béléné est l'Alcatraz du Danube. Une île de quarante-huit hectares qui hébergea les prisonniers politiques de 1949 à 1962. Aujourd'hui mille pensionnaires de droit commun y séjournent. [...] Les surveillants veulent me présenter leurs prisonniers modèles 'parce que la plupart sont analphabètes et ne parlent pas très bien le bulgare. Soixantedix pour cent d'entre eux sont des Tziganes'. Je refuse et m'adresse aux prisonniers selon mon inspiration. (Graff, 1998: 74)

De même, l'auteur du Bagne des Hä̈doucks refusait en 1932 de servir de caution journalistique à des modes d'incarcération échappant de toute façon aux normes occidentales, puisqu'à cette époque la Roumanie connaissait une réforme de son système judiciaire.

Le parcours routier de l'équipe franco-allemande, lié aux multiples points d'observation qu'offre le fleuve, rend aussi compte d'une guerre d'influence au cœur des préoccupations relevant d'une défense militaire mondialisée, au-delà de stratégies pour la conservation de la planète.

À Kozloduj, km 720, se trouve la deuxième centrale atomique danubienne, la première se trouvant à Cernavoda, en Roumanie. En 1995, elle s'illustra dans la presse mondiale par plusieurs fuites radioactives. Les autorités se firent prier pour nous accorder l'autorisation de filmer. Qui s'en étonnera? On n'entre pas non plus dans une centrale française comme dans un moulin. (Graff, 1998: 78)

Béléné n'est nullement évoquée (ce qu'elle est aujourd'hui) comme un lieu d'implantation nucléaire offrant aussi peu de garanties de sécurité que Kozloduj. Le lieu est en revanche remarqué pour sa beauté. On songe à la mythique Ada-Kaleh célébrée jusque dans le folklore roumain comme le lieu d'isolement d'une jeune tisseuse ottomane condamnée par une grave maladie. Ce paradis fiscal du sultan Ali-Kadri commémoré par un reportage de 1936 (Brunea-Fox, 1979: 246-262) a été aussi l'objet d'un texte nostalgique de Mircea Cărtărescu, brillant écrivain roumain des années 80 , dans une anthologie explicitement consacrée au naufrage des frontières de l'ancienne Europe (Raabe et Sznajderman : 2007). Ada-Kaleh, submergée en 1970 en raison de la construction de la centrale hydro-électrique des Portes de Fer, mesurait 1,75 sur 0,5 km. D’autres îles fluviales sont signalées par M. Graff pour leur attrait de sites invitant à la croisière.

Après Kozloduj, le voyageur découvre de magnifiques îles avec des bancs de sable et des oasis dignes du Sahara. Elles bougent comme des dunes en fonction de la hauteur des eaux. Certaines sont nues et brillent comme des 
diamants au soleil. D’autres sont recouvertes par des touffes de végétation. Elles sont le refuge de milliers d'oiseaux qui adorent se réfugier dans les jardins secrets du fleuve. (Graff, 1998: 79)

La réactivation de la peur de la guerre, des risques de pollution, de contamination nucléaire mis en balance avec les invites à la cupidité suscitées par la soudaine ouverture de territoires jusque là censurés, fait bien partie des thèmes "économiques» débattus par le livre de M. Graff. Ces motifs répondent à la mise en garde contre une "poudrière balkanique» pratiquée, à tort ou à raison, au début $\mathrm{du} \mathrm{xx}^{\mathrm{e}}$ siècle comme suite aux déplacements de voyageurs occidentaux dans ces régions, contribuant à une valorisation du reportage de voyage comme genre littéraire contemporain de l'urgence, dédouané des stigmates de la futilité.

\section{Bibliographie}

Blairy, Jean, 1946, Crépuscule danubien - Yougoslavie-Roumanie. Paris, Librairie Plon. Bourcier, Emmanuel, 1932, Le bagne des haïdoucks - Reportage, Paris, Ed Baudinière. Brunea-Fox, Filip, 1979, Reportajele mele - 1927-1938. Cuvint înainte, note, antologie și ediție îngrijită de Lisette Daniel-Brunea, Bucuresti. Editura Eminescu.

Claretie, Léo, 1913, Feuilles de route en Roumanie - A travers le pays, Paris, Editions Sansot.

Durandin, Catherine, avec la collaboration de Magda Cârneci, 2004, Perspectives roumaines: du post-communisme à l'intégration européenne, Paris, L'Harmattan.

Fabritius, Aurora, 2008 (editor in chief), The Danube Delta. "Plural" - Culture and Civilization, Bucharest, The Romanian Cultural Institute.

Fernandez, Dominique, 1998, Rhapsodie roumaine, Paris, Grasset.

Graff, Martin, 1998, Le réveil du Danube - Géopolitique vagabonde de l'Europe, Strasbourg, La Nuée Bleue/ DNA.

Magris, Claudio, 1986, 1988, Danube. Traduit de l'italien par Jean et Marie- Noelle Pastureau, Paris, Folio n 2162.

Magris Claudio, 1999, 2001, Utopie et désenchantement. Traduit de l'italien par Jean et Marie-Noelle Pastureau, Paris, Gallimard.

Monneyron Frédéric, Thomas Joël (dir.), 2005, Automobile et littérature. Publié avec le concours du centre de recherches VECT (Voyages, Echanges, Confrontations, Transformations), Presses universitaires de Perpignan, Collection «Études».

Orient (D’) José, 1932, Arc-en-ciel balkanique, Paris, Ed. Victor Attinger.

Raabe, Katherine, Sznajderman Monika (dir.), 2007, Last and Lost. Atlas d'une Europe fantôme. Récit de voyage (treize auteurs traduits balkaniques et centreeuropéens traduits), Ed. Noir sur Blanc. 
Stahl, Paul, Henri, 2004, Appartenance ethnique et confession religieuse, Exemples du Sud-Est européen. Etudes et documents balkaniques et méditerranéens $n^{\circ} 27$, Paris, Bibliothèque Nationale, ISSN 1269-1720.

Todorov, Tzvetan, 1996, L’homme dépaysé, Paris, Ed. du Seuil.

Todorov, Tzvetan, 2002, Devoirs et délices, Une vie de passeur - Entretiens avec Catherine Portevin, Paris, Ed. du Seuil.

Verne, Jules, 1988, 2002, (Société Jules Verne, première édition à tirage limité) Le Beau Danube Jaune, Paris, Folio n 3719. 